\title{
Laser-induced dispersion control
}

\author{
Gennady Rasskazov, Anton Ryabtsev, Vadim V. Lozovoy, and Marcos Dantus* \\ Department of Chemistry, Michigan State University, East Lansing, Michigan 48824, USA \\ ${ }^{*}$ Corresponding author: dantus@msu.edu
}

Received March 12, 2014; revised April 2, 2014; accepted April 7, 2014; posted April 8, 2014 (Doc. ID 208163); published 0 MONTH 0000

\begin{abstract}
An intense laser pulse is used to control the spectral phase of a weak probe pulse as they overlap in fused silica. The laser-induced linear chirp is controlled by the delay time between pulses. Dependence from intensity and spectral phase of the pump pulse is also studied. Experimental data is validated by numerical simulation based on optical Kerr effect. Results show that laser-induced pulse shaping is possible and may be useful for intracavity pulse compression and shaping in enhancement cavities. (c) 2014 Optical Society of America

OCIS codes: (320.5540) Pulse shaping; (190.3270) Kerr effect; (160.6030) Silica; (120.5060) Phase modulation; (320.0320) Ultrafast optics.

http://dx.doi.org/10.1364/OL.99.099999
\end{abstract}

Femtosecond lasers continue to permeate scientific research and applications because of their ability to deliver high peak powers and, hence, facilitate nonlinear optical processes. The pulse duration and peak intensity of femtosecond lasers is closely dependent on their spectral phase. To compensate for linear chirp, prism/grating compressors [1,2], or chirped mirrors [3] , have been used. Correction of high-order dispersion has been preferentially carried out using pulse shaper-based measurement and correction, reaching sub-two cycle pulse durations [는 $\underline{6}$ ]. Recent work from our group found that, in addition to the group delay dispersion (GDD) experienced by femtosecond lasers, intense femtosecond lasers $\left(>10^{11} \mathrm{~W} / \mathrm{cm}^{2}\right)$ undergo a nonlinear laser-induced GDD, which can be normal or anomalous depending on the chirp of the input pulse, and was found to be several times larger than the static dispersion [7].

Here, we explore the ability of an intense pulse to control the dispersion experienced by a probe pulse taking advantage of the optical Kerr effect (OKE) and, in particular, what is known as cross-phase modulation [8]. Of particular interest is the spectral phase imprinted on the output pulses. One can envision a setup, such as that used in an enhancement cavity to create high harmonic generation [9], where part of the input laser could be used to control the intracavity dispersion and optimize the conversion efficiency. This approach is related to the proposal and eventual demonstration of phase modulation through the use of a pump pulse, used to align molecules in the gas phase and thereby causing a change in the refractive index, which in turn modulates the phase of a time-delayed probe pulse $[\underline{10}, 11]$.

Measurements of laser-induced GDD presented here are confirmed by two different experimental approaches and simulated through use of the OKE theory. To register single-shot time-resolved GDD measurements, we used real-time multiphoton intrapulse interference phase scan (RT-MIIPS) [12], which is based on the predictable changes in the second-harmonic spectrum of femtosecond lasers as a function of spectral phase [6]. The results obtained are validated by the Fourier-transform spectral interferometry (FTSI) method $[\underline{13}, 14]$. From the FTSI data we extracted GDD values. Our measurements differ from traditional OKE measurements, where the OKE phase shift $[\underline{15,16]}$, or nonlinear absorption and refraction are obtained [17]. The spectral phase shift induced through the OKE is relatively small (less than $1 \mathrm{rad}$ ); however, the GDD (second derivative of the phase with respect to frequency) is large enough to be useful for pulse compression.

To illustrate this principle, we start with an intensitydependent correction to the refractive index of the media in time domain:

$$
n(t)=n_{0}+n_{2} I(t)
$$

where $n_{0}$ is the weak-field refractive index at carrier frequency, $n_{2}$ is the nonlinear refractive index of media, $I(t)$ is an intensity profile of the pulse, and higher-order terms are neglected. If pump and probe pulses have mutually orthogonal polarization, then the probe beam experiences $2 / 3$ of the nonlinear refractive index induced by the pump pulse [18]. This relationship is related to the cross-phase modulation phenomenon. The phase shift experienced by probe pulse in the time domain is:

$$
\Delta \phi(t)=-\frac{2}{3} \cdot n_{2} I_{\text {pump }}(t) L \omega_{0} / c,
$$

where $\omega_{0}$ is the carrier frequency, $L$ is the length of the medium, $I_{\text {pump }}(t)$-intensity profile of pump pulse.

The pump-induced phase change model is illustrated in Fig. 1, where the probe pulse is illustrated by the black curve and the pump-induced phase distortion in the sample at different pump-probe delay times is depicted by a red curve. The function describing the phase distortion is broader, since it depends on the crossing angle between

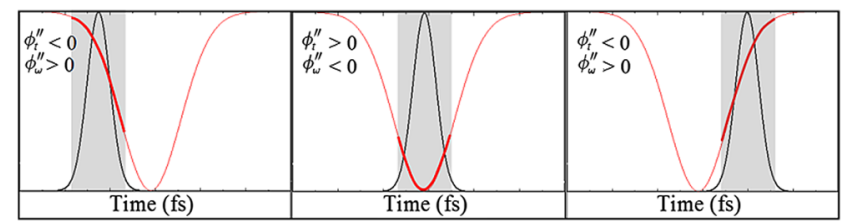

Fig. 1. Schematic illustration of the OKE-induced GDD (not to scale, pump peak intensity is a few orders of magnitude greater than probe) for three pump-probe relative positions at negative, $0 \mathrm{fs}$, and positive time delays. Intensity profile of the probe pulse (black curve) moves along the OKE phase distortion function (red line). At different delay times, the sign and magnitude of the GDD $\left(\varphi_{\omega}^{\prime \prime}\right)$ changes. 
the beams and dispersion of the medium. Note that the second derivative of the phase in the time domain $\left(\varphi_{t}^{\prime \prime}\right)$ has the opposite sign to that in the frequency domain $\left(\varphi_{\omega}^{\prime \prime}\right)[19]$.

The electric field of the probe pulse at the exit of the sample is:

$$
\begin{aligned}
E(t)= & E_{0} e^{-t^{2} / 2 \tau_{0, \text { probe }}^{2}} \\
& \times \exp \left[i \omega_{0} t-i\left(n_{0}+2 / 3 * n_{2} I_{\text {pump }} e^{-t^{2} / \tau_{0, p u m p}^{2}}\right) \frac{L \omega_{0}}{c}\right],
\end{aligned}
$$

where $E_{0}=\sqrt{I_{0}}$ is the amplitude of the field of the probe; $I_{0}$ is the intensity of the probe; $\tau_{0, \text { probe }}, \tau_{0, \text { pump }}$ are the fullwidth at half-maximum (FWHM) duration of probe and pump pulses, correspondingly; and $I_{\text {pump }}$ is the intensity magnitude of the pump pulse. We used the first two terms of the Taylor expansion for the exponential function in the phase, and Fourier-transformed it to the frequency domain to obtain GDD for small phase distortions of less than 1 radian:

$$
\phi_{\omega}^{\prime \prime} \approx \frac{2 \tau_{0, \text { probe }}^{2} \cdot \phi_{2}}{1+4 \phi_{2}^{2}},
$$

where $\phi_{2}=-2 / 3 * n_{2} I_{\text {pump }} L \omega_{0} / c$ is a maximum of nonlinear phase shift.

The pump-probe experimental setup is shown in Fig. 2. The laser system comprises a Ti:sapphire oscillator (KMLabs), MIIPSBox640 pulse shaper (BioPhotonic Solutions, Inc.), and a regenerative amplifier (SpectraPhysics, Spitfire, $800 \mathrm{~nm}$ central wavelength, $1 \mathrm{kHz}$ rep. rate). Pulses are compressed to their transform-limited duration of $40 \mathrm{fs}$ using the MIIPS technique [4-6,20]. The laser is split into two beams with orthogonal linear polarizations, denoted in the schematics as "pump" and "probe." The probe beam is sent through a computercontrolled delay line. After passing through a focusing lens, the beams are overlapped at a $4^{\circ}$ incidence angle on a $1.8 \mathrm{~mm}$ thick fused quartz sample, placed a few centimeters away from the lens focus. The intensity of the pump beam on the sample is varied in a range of $0.3 \times$ $10^{12}-1.7 \times 10^{12} \mathrm{~W} / \mathrm{cm}^{2}$ by adjusting a variable neutral density filter. The probe beam intensity is a few orders of magnitude lower than that of the pump.

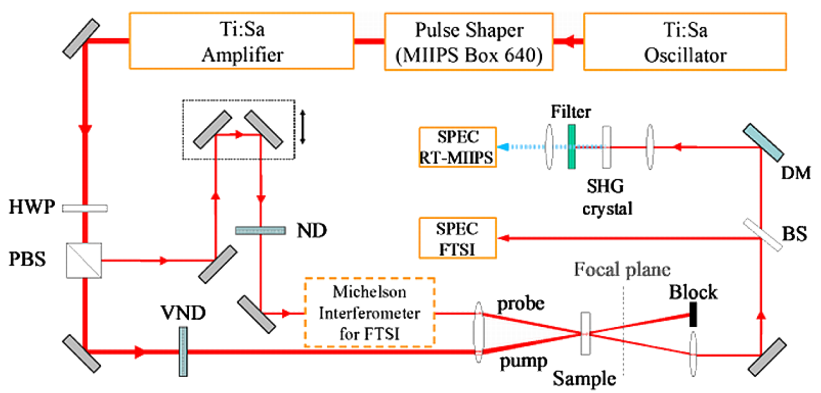

Fig. 2. Experimental setup for transient dispersion measurements via RT-MIIPS and FTSI methods. The Michelson interferometer is used only for FTSI. HWP, half-wave plate; PBS, polarizing beam splitter; VND, variable neutral density filter; ND, neutral density filter; DM, dielectric mirrors.
For RT-MIIPS calibration and measurements [12], the probe beam, after transmitting through the sample, is reflected by a set of dielectric mirrors that introduce a static spectral phase dominated by third-order dispersion (TOD) of $-150,000 \mathrm{fs}^{3}$. The conditioned probe beam is then focused on a second harmonic generation (SHG) crystal and the SHG spectrum is recorded with a fibercoupled compact spectrometer (Ocean Optics HR4000). There is no contribution into SHG from the stray pump light because pump and probe have perpendicular polarization. For the RT-MIIPS calibration process, we apply the procedure using only the probe beam through the sample. For pump-probe measurements, the pump beam is unblocked and the SHG spectrum is acquired for each pump-probe delay position.

For FTSI measurements, a Michelson interferometer is used to make two replicas of the probe pulse with $1 \mathrm{ps}$ time separation. The pump pulse arrives at the sample after the first reference probe replica so that the pump-induced changes affect only the second replica. These distortions lead to a phase change between the two replicas. The spectral interferometry signal collected by a spectrometer (Ocean Optics QE65000) bears these changes. The GDD values for each pump-probe delay position are extracted by Fourier transformation and polynomial fitting of the retrieved phase.

RT-MIIPS measures the second derivative of spectral phase directly. The raw experimental data is a $2 \mathrm{D}$ spectrogram of SHG signal as a function of the pumpprobe delay [12]. For each single SHG spectrum from this spectrogram, a maximum position is calculated. Through one-to-one correspondence between SHG peak position and second derivative of spectral phase, the GDD can be extracted. The corresponding induced GDD values experienced by the probe pulse at each time delay value are plotted as a function of delay time in Figs. 3(a)-3(c).

Experimental results in Fig. 3(a) show negative GDD around zero delay time with the minimum value of $-139 \mathrm{fs}^{2}$. There is a subsequent rise of GDD at about $100 \mathrm{fs}$ delay time, with the maximum of $+70 \mathrm{fs}^{2}$. These data were obtained with a pump peak intensity of $0.68 \times 10^{12} \mathrm{~W} / \mathrm{cm}^{2}$. The accuracy of the GDD measurements is within $\pm 20 \mathrm{fs}^{2}$, as determined during the calibration procedure using a calibrated pulse shaper [12]. The FTSI measurements [see the blue squares in Fig. 3(a)] closely reproduce the RT-MIIPS results in shape and magnitude. The FTSI curve was shifted vertically by $10 \mathrm{fs}^{2}$ during the data processing. The observed nonzero GDD offset originates from the unbalanced Michelson interferometer.

The theoretical curve showed in Fig. 3(a) (black solid line) is a result of numerical simulation based on Fourier transformation of Eq. (3). We assumed a Gaussian profile for the pump-induced phase with FWHM of 148 fs. The majority of the broadening is caused by the crossing angle between the pulses. The asymmetric shape of the experimental GDD profile is caused by the optical response of the media through SPM [21] and the nuclear response [22]. To reach quantitative agreement between model and experiment, we introduce a 1.85/3 factor for the nonlinear phase shift. This value is reasonably close to the theoretically expected value $2 / 3$ [18]. The mismatch between theoretical and experimental values is likely from 

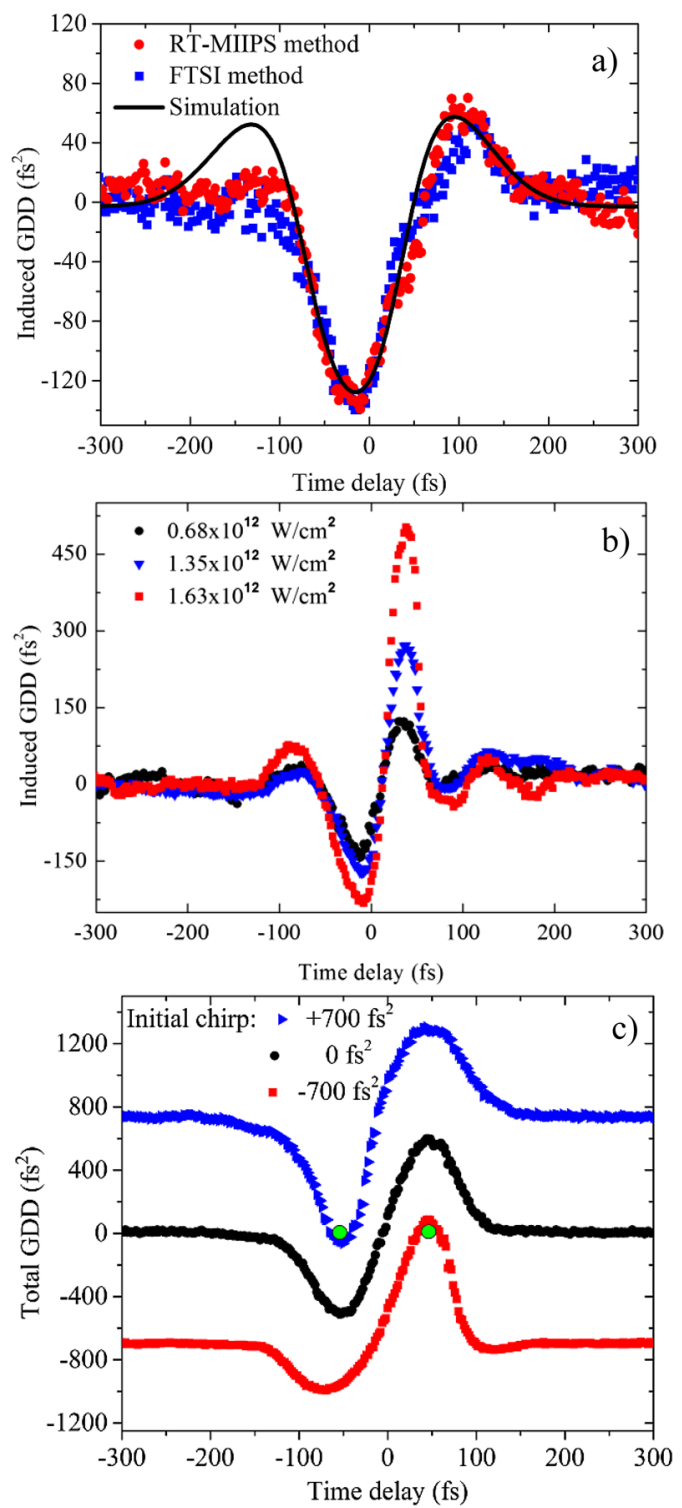

Fig. 3. (a) Experimental measurement and simulation of laserinduced GDD acquired by the probe pulse with respect to pump-probe delay time. Red and blue curves are experimental data from RT-MIIPS and FTSI methods, respectively. Black solid curve is the result of OKE simulation model. (b) GDD as a function of pump-probe delay time for different values of a pump peak intensity. (c) GDD as a function of pumpprobe delay time for different values of initial chirp. Black circles for $1.7 \times 10^{12} \mathrm{~W} / \mathrm{cm}^{2}$, red squares and blue triangles for $1.15 \times 10^{12} \mathrm{~W} / \mathrm{cm}^{2}$.

the cumulative uncertainty on the intensity measurements, which depend on average power, pulse duration, and beam size.

Figures 3(b) and 3(c) show the dependence of pumpinduced GDD with respect to peak intensity and initial chirp value, correspondingly. From Fig. 3(b), it follows that, as the intensity of the pump laser increases, the GDD magnitude increases in both negative and positive GDD regions. In the case of the red curve (squares) the GDD has it minimum $-232 \mathrm{fs}^{2}$ and maximum $+502 \mathrm{fs}^{2}$, values that are several times the corresponding GDD of fused silica with the same $1.8 \mathrm{~mm}$ thickness $\left(65.16 \mathrm{fs}^{2}\right)[5]$.
The observed oscillations [Fig. 3(b), red squares] for positive pump-probe delay times are similar to those reported in fused silica when looking at Kerr gate signals [22], which assign the oscillations to the nuclear contribution (phonons) to the signal. Figure 3(c) shows the dependence between induced GDD and initial chirp value. The green dots in Fig. 3(c) indicate the time delays where total GDD is zero, meaning that the induced GDD cancels the chirp of the input probe pulse and, therefore, compresses it to TL duration. Figure 3(c) has evidence of the two-beam coupling process [23]. The energy transfer between pump and probe [24] beams is proven by the asymmetric shape of red (squares) and blue (triangles) curves in Fig. 3(c). Changes in the overall width for the different measurements shown in Figs. 3(a)-3(c), are caused by changes in the crossing angle between pump and probe pulses. Narrower widths require near collinear geometry.

The measurements presented here are relevant to pump-probe experiments carried out in fused silica cuvettes; femtosecond laser sources involving supercontinuum generation contained in hollow waveguides [25,26]; ultrafast fiber lasers that combine high peak intensity and long propagation length; and silicon-based high-speed on-chip devices [27]. Finally, it has become of interest to use enhancement cavities to create high harmonic generation [9]. The efficiency of such cavities depends on intracavity dispersion, which is managed through the use of chirped mirrors and prisms. Although a deformable mirror and a prism could be used to control intracavity dispersion [28], one could consider using the concept described here; taking advantage of the interaction between two pulses in a nonlinear medium to control dispersion in an enhancement cavity.

In summary, the laser-induced GDD acquired by a probe pulse in fused silica as a result of interaction with an intense pump laser through changes in the refractive index of media has been measured. We show that the GDD can be tuned in the range of hundreds of $\mathrm{fs}^{2}$, both negative and positive, by changing the time delay between pulses. The induced GDD magnitude and sign depends not only on the intensity of the pump pulse but also on the initial chirp. A numerical model, based on the OKE, is used to provide a theoretical foundation for the experimental results. Our findings could be used to design a pulse shaping method that exploits the nonlinear interaction between strong and weak laser pulses inside a Kerr medium, to control the temporal profile of the weak pulse through cross-phase modulation. This type of phase control could be important in enhancement cavities used for producing higher harmonics or in a cavity where the space appears to be a limiting factor, and where other stretcher/compressor devices would not be suitable to use.

This work was funded in part by CHE-1014538 Early-Concept Grant for Exploratory Research from the NSF. Tanja Pietraß program officer is gratefully acknowledged.

\section{References}

1. R. L. Fork, O. E. Martinez, and J. P. Gordon, Opt. Lett. 9, 150 (1984). 
2. D. Strickland and G. Mourou, Opt. Commun. 56, 219 (1985).

3. R. Szipöcs, K. Ferencz, Ch. Spielmann, and F. Krausz, Opt. Lett. 19, 201 (1994).

4. V. V. Lozovoy, I. Pastirk, and M. Dantus, Opt. Lett. 29, 775 (2004).

5. B. Xu, J. M. Gunn, J. M. Dela Cruz, V. V. Lozovoy, and M. Dantus, J. Opt. Soc. Am. B 23, 750 (2006).

6. Y. Coello, V. V. Lozovoy, T. C. Gunaratne, B. Xu, I. Borukhovich, Ch. Tseng, T. Weinacht, and M. Dantus, J. Opt. Soc. Am. B 25, A140 (2008).

7. G. Rasskazov, A. Ryabtsev, D. Pestov, B. Nie, V. V. Lozovoy, and M. Dantus, Opt. Express 21, 17695 (2013).

8. M. N. Islam, L. F. Mollenauer, R. H. Stolen, J. R. Simpson, and H. T. Shang, Opt. Lett. 12, 625 (1987).

9. R. J. Jones, K. D. Moll, M. J. Thorpe, and J. Ye, Phys. Rev. Lett. 94, 193201 (2005).

10. V. Kalosha, M. Spanner, J. Herrmann, and M. Ivanov, Phys. Rev. Lett. 88, 103901 (2002).

11. R. A. Bartels, T. C. Weinacht, N. Wagner, M. Baertschy, C. H. Greene, M. M. Murnane, and H. C. Kapteyn, Phys. Rev. Lett. 88, 013903 (2001).

12. D. Pestov, A. Ryabtsev, G. Rasskazov, V. V. Lozovoy, and M. Dantus, Opt. Eng. 53, 051511 (2014).

13. E. Tokunaga, A. Terasaki, and T. Kobayashi, Opt. Lett. 17, 1131 (1992).

14. L. Lepetit, G. Chériaux, and M. Joffre, J. Opt. Soc. Am. B 12 , 2467 (1995).

15. E. Tokunaga, A. Terasakiy, and T. Kobayashi, J. Opt. Soc. Am. B 12, 753 (1995).
16. P. Martin, S. Guizard, Ph. Daguzan, G. Petite, P. D'Oliveira, P. Meynadier, and M. Perdrix, Phys. Rev. B 55, 5799 (1997).

17. M. Sheik-Bahae, A. A. Said, T.-H. Wei, D. J. Hagan, and E. W. Van Stryland, IEEE J. Quantum Electron. 26, 760 (1990).

18. G. I. Stegeman, Contemporary Nonlinear Optics, G. P. Agrawal and R. W. Boyd, eds., (Academic, 1992), pp. 16-18.

19. A. M. Weiner, Ultrafast-Pulse Measurement Methods in Ultrafast Optics (Wiley, 2009), pp. 112-118.

20. V. V. Lozovoy, I. Pastirk, K. A. Walowicz, and M. Dantus, J. Chem. Phys. 118, 3187 (2003).

21. L. Rapp, HAL: hal-00301019, version 1 (2008), http://hal .archives-ouvertes.fr/hal-00301019.

22. K. Dota, J. A. Dharmadhikari, D. Mathur, and A. K. Dharmadhikari, Appl. Phys. B 107, 703 (2012).

23. S. Smolorz and F. Wise, J. Opt. Soc. Am. B 17, 1636 (2000).

24. Q. Yang, G. Xu, X. Liu, J. Si, and P. Ye, Appl. Phys. B 66, 589 (1998).

25. M. Nisoli, S. Stagira, S. De Silvestri, O. Svelto, S. Sartania, Z. Cheng, M. Lenzner, C. Spielmann, and F. Krausz, Appl. Phys. B 65, 189 (1997).

26. G. Stibenz, N. Zhavoronkov, and G. Steinmeyer, Opt. Lett. 31, 274 (2006).

27. A. R. Motamedi, A. H. Nejadmalayeri, A. Khilo, F. X. Kärtner, and E. P. Ippen, Opt. Express 20, 4085 (2012).

28. N. K. Metzger, W. Lubeigt, D. Burns, M. Griffith, L. Laycock, A. A. Lagatsky, C. T. A. Brown, and W. Sibbett, Opt. Express 18, 8123 (2010). 